\title{
Nicht nur die Seeversicherung. Philipp Hellweges Projekt zur Geschichte des Versicherungsrechts
}

\author{
Albrecht Cordes
}

Online publiziert: 3. Februar 2020

(C) Der/die Autor(en) 2020

Welch ein Auftakt! Mit drei Büchern auf einmal präsentiert der Augsburger Wirtschaftsrechtler und Rechtshistoriker Phillip Hellwege sein großes Forschungsfeld, die Geschichte des Versicherungsrechts. Zwei Sammelbände und eine Monografie, im Sommer 2018 fast gleichzeitig erschienen, werden dem Projekt die gebührende Aufmerksamkeit sichern. Die Forschungsmittel entstammen dem „Horizon 2020“Programm des European Research Council ERC, von dem Hellwege einen „Consolidator Grant" erhalten hat. Mit diesen Mitteln finanziert er sein Vorhaben „Comparative History of Insurance Law in Europe (CHILE)“. Die Ergebnisse erscheinen in der neuen Schriftenreihe „Comparative Studies in the History of Insurance Law Studien zur vergleichenden Geschichte des Versicherungsrechts (HIL)““. Deren erste drei Titel sind ein Überblicksband über die Versicherungsgeschichte (HIL Band 1) und zwei Bücher über das höchst merkwürdige Rechtsinstitut der Tontinen.

Band 1 enthält acht Länderberichte nebst Einleitung und Zusammenfassung; zwei knappe Querschnittsreferate aus sozial- und wirtschaftsgeschichtlicher Sicht beschließen den Band. Die Berichte setzen sich zu einer chronologischen Reise durch das südliche, westliche und nördliche Europa zusammen. Auf das Mittelmeer (Italien - Frankreich - Spanien) folgen gleich drei Beiträge zu Belgien und den Niederlanden, wodurch hier ein Schwerpunkt der Darstellung entsteht. Berichte über England und Schottland, Deutschland und Skandinavien schließen sich an. Die

A Comparative History of Insurance Law in Europe. A Research Agenda, ed. by Phillip Hellwege; The Past, Present, and Future of Tontines. A Seventeenth Century Financial Product and the Development of Life Insurance, ed. by Phillip Hellwege; Phillip Hellwege, A History of Tontines in Germany. From a multi-purpose financial product to a single-purpose pension product (Comparative Studies in the History of Insurance Law - Studien zur vergleichenden Geschichte des Versicherungsrechts, hg. von Phillip Hellwege, Bde. 1-3) Berlin: Duncker \& Humblot 2018.

\footnotetext{
A. Cordes $(\triangle)$

Fachbereich Rechtswissenschaft, Johann Wolfgang Goethe-Universität, Frankfurt am Main, Deutschland

E-Mail: cordes@jur.uni-frankfurt.de
} 
besonders gelungenen Beiträge von Mauro Fortunati (Italien) sowie Dirk Heirbaut und Dave De ruysscher (Belgien) seien hervorgehoben.

Die vom Herausgeber Hellwege vorgegebene Frage ist das Verhältnis der Seeversicherung zu anderen Versicherungstypen, vor allem jenen auf Gegenseitigkeit, unter denen die Feuerversicherung eine frühe wichtige Rolle gespielt hat. Entstammen die ältesten Wurzeln des Versicherungsrechts allein der Seeversicherung? Oder sind die verschiedenen Formen von Beistandsvereinbarungen auf Gegenseitigkeit eine gleichwertige, unabhängig danebenstehende Quelle? Das sind die beiden Pole, zwischen denen die jeweiligen nationalen Diskurse oszillieren; vor allem in Deutschland betont die bisherige Literatur gern die seit der Karolingerzeit überlieferten Beistandsversprechen als unabhängige zweite Wurzel.

Reizvoll ist es, dass die Beiträger sich alle auf den beiden Ebenen der historischen und der historiographischen Untersuchung bewegen. Letzteres rechtfertigt auch die Einteilung der Berichte anhand der heutigen Grenzen, was für eine rein historische Untersuchung nur begrenzt sinnvoll wäre, da sich beispielsweise in Brügge, Antwerpen und Amsterdam intensive, nicht an modernen Grenzen endende Begegnungen und gegenseitige Beeinflussungen der internationalen Prinzipien beobachten lassen oder in Italien nicht von „dem“ italienischen Versicherungsrecht gesprochen werden kann, weil dort jede der großen Stadtrepubliken ihre eigenen Prinzipien entwickelte.

Die historiographischen sind zugleich nationale Diskurse. Das ist ein vielleicht nicht überraschender, aber trotzdem eindrücklich belegter Befund des Bandes und auch des ganzen Forschungsvorhabens: Bis heute wirken die aktuellen nationalen Grenzen als Scheuklappen: die einschlägigen Studien in den Nachbarländern werden nur selektiv zur Kenntnis genommen. Dabei ist es jedenfalls bei den Seeversicherungen eine besonders interessante Frage, wann sie aus den lokalen (venezianischen, genuesischen usw.) Anfängen herauswachsen und zu internationalen Phänomenen werden.

Der Band HIL 2 beschäftigt sich mit Vergangenheit, Gegenwart und Zukunft der Tontinen, einem Rechtsinstitut, von dem der Rezensent auch nach der Lektüre noch nicht ganz sicher ist, worum es sich genau handelt. Im Zentrum des Bedeutungsfelds herrscht noch Klarheit. Es geht um eine Verabredung, bei welcher der Veranstalter am Beginn der Unternehmung von allen Teilnehmern eine Kapitalsumme erhält, die dauerhaft bei ihm verbleibt. Als Gegenleistung zahlt er dafür allen Teilnehmern eine jährliche Leibrente, z. B. $5 \%$ ihres Kapitals. Die Pointe ist die folgende: Wenn die Teilnehmer nach und nach sterben, werden die Raten der Verstorbenen den Überlebenden zugeschlagen. Deren Rente steigt also, bis schließlich der letzte Überlebende die Anteile aller Teilnehmer seiner Tontine erhält.

Manches daran ist kurios, angefangen mit dem dubiosen Namenspatron, einem gewissen Hochstapler namens Lorenzo Baroncini aus Rom, Sohn eines Gärtners und einer Wäscherin, der mit Informationen handelte und aufstieg, die er sich durch Spionage und auf anderen anrüchigen Wegen beschaffte. Er usurpierte den vornehmen Namen Tonti - manchmal auch adlig „de Tonti“ -, musste aus Italien nach Frankreich fliehen, schaffte es dort, als angesehener neapolitanischer Bankier und ehemaliger Gouverneur von Gaeta durchzugehen. Die hier referierte, wenig freundliche Variante seiner Vita stützen Riesch/Gallais-Hamonno (S. 19-47) allerdings auf den Bericht des mit Tonti verfeindeten Zeitgenossen Camillo Tutini. Vielleicht 
handelt es sich also doch nicht um Hochstapelei, sondern um üble Nachrede. Tonti passt aber jedenfalls mit dieser nebulösen Vita gut in das halbseidene Milieu von kreativen Finanzjongleuren, die mit den unterschiedlichsten Mitteln die nach 1648 maroden Kassen der Fürstentümer zu füllen versprachen. Es gelang manchen dieser Figuren tatsächlich, das Ohr einflussreicher Finanzminister zu gewinnen. Tonti etwa erlangte Zutritt zu Kardinal Mazarian am Hofe Ludwigs XIV. Doch drei Projekte, erste Tontinen durchzuführen, scheiterten, und erst nach Tontis Tod organisierte die französische Krone einige erfolgreiche Tontinen.

Das erste nach dem oben skizzierten Muster organisierte Geschäft fand aber schon 1657 in Danzig statt. Auch die Niederländer wurden früh aktiv, und vor der Wende zum 18. Jh. hatten in vielen europäischen Ländern derartige Veranstaltungen stattgefunden. Daher ist durchaus nicht klar, wie groß Tontis eigener Anteil an dem Finanzinstrument, das seinen Namen trägt, tatsächlich war. Der Sammelband HIB 2 geht die Tontinen nach drei einleitenden Beiträgen Land für Land durch, ist also ähnlich organisiert wie Band 1. Am Schluss stehen vier Beiträge über Gegenwart und (die vermutlich nicht sehr strahlende) Zukunft der Tontinen und noch einmal zwei vergleichende Zusammenfassungen. Wiederum ist der Herausgeber zugleich der fleißigste Autor und sorgt durch gut gegliederte Einleitungs- und Schlusskapitel dafür, dass dem Leser stets klar ist, welche Rolle die einzelnen Beiträge in seinem Gesamtkonzept spielen. Nur selten begegnen Sammelbände, die so stringent durchorganisiert sind.

Der Band schwankt ein wenig zwischen Wort- und Begriffsgeschichte, weil er sich einerseits primär an den Ausschreibungen orientiert, die sich selbst „Tontinen“ nennen, andererseits frühe Ausschreibungen einbezieht, die noch nicht „,Tontinen" heißen, und andererseits manche Tontinen wegen struktureller Unterschiede als „,misnomers“ ausblenden will. Interessant ist etwa, dass der Terminus heute in Afrika und Ostasien begegnet und dort Kleinkreditgemeinschaften im informellen Sektor bezeichnet, bei denen alle Mitglieder einzahlen und dann der Reihe nach Existenzgründungskredite an sie vergeben werden. Methodisch problematisch ist dieses Vorgehen trotzdem, weil auch innerhalb dessen, was sich nach Hellwege Tontine nennen ,darf', der Spielraum der Gestaltungsmöglichkeiten ziemlich groß ist. Wo hier die Grenze gezogen wird, ist nicht ganz klar.

Die Vielfalt der verfolgten Interessen und Gestaltungsmöglichkeiten wird besonders in dem Band HIL 3 deutlich: Hellweges Monografie über die deutschen Tontinen. In Deutschland lassen sich nämlich die deutlichsten Indizien für eine Antwort auf die Hauptfrage des Bandes HIL 2 finden. Der hatte sich das Ziel gesetzt, den Einflüssen der Tontinen auf das Recht der Lebensversicherungen nachzuspüren, das in der versicherungsrechtlichen Literatur häufig behauptet, aber nie recht bewiesen wurde. Dazu gehörten die Berücksichtigung des Alters der Teilnehmer, auf das in den frühen Tontinen noch keine Rücksicht genommen worden war, die Aufstellung und Berechnung von Sterbetafeln, die Kappung der exorbitant hohen Ansprüche der letzten Überlebenden und weitere Modifizierungen. Rechtshistorisch ist der Band HIL 3 vor allem deshalb interessant, weil Hellwege die Quellenbasis für deutsche Tontinen erheblich verbreitern kann. Diesen Quellenfunden widmet er die erste Hälfte des Buchs (Teil C, S. 35-106). Während man bisher allenfalls von rund zwei Handvoll Tontinen wusste und sich dabei vor allem auf das 17. Jahrhundert konzentrierte, 
kann Hellwege nun nicht weniger als 48 von Staaten, Provinzen und Kommunen und sechs weitere von Pensionsfonds veranstaltete Tontinen präsentieren. Bei der ersten Gruppe liegt der Schwerpunkt in der 2. Hälfte des 18. Jahrhunderts, die Tontinen der Pensionsfonds folgen etwas später und stammen aus der Zeit von 1778-1838. Diese gehören nach Hellwege einer eigenen, zweiten Periode der Geschichte der Tontinen an. Eine dritte Phase folgt im späten 19. Jh. (bis 1905), als amerikanische Versicherungsgesellschaften ein Kombinationsprodukt aus Lebensversicherung und Tontine mit Erfolg in Europa vermarkten.

Hellwege sieht in dieser 2. und 3. Phase auf der Seite der Kunden eine Verengung auf ein konkretes Interesse, nämlich die Sicherung einer Pension. So ist der Untertitel „From a multi-purpose financial product to a single-purpose pension product“" gemeint. Das trifft vermutlich zu, denn über die Gründe der Zeichner in der Anfangs- und Blütezeit der Tontinen kann man oft nur spekulieren. Es lassen sich Motive erahnen, die denen der Teilnehmer an den beliebten Wetten, Lotterien und Spekulationen des 17./18. Jahrhunderts ähneln: Wer am längsten lebt, gewinnt auch finanziell. Aber der Versorgungsaspekt, der auch schon den seit dem Mittelalter weit verbreiteten Rentengeschäften zugrunde lag, dürfte ebenfalls eine Rolle gespielt haben, zumal man nicht nur sich selbst, sondern auch einen dritten Begünstigten in die Tontine einkaufen konnte.

Auf der Seite der Veranstalter ist wohl in erster Linie zwischen der öffentlichen Hand, die fiskalisch interessiert war, weil sie bei erfolgreichen Tontinen mit einem Schlag ansehnliche Geldbeträge einnahm, und privaten Unternehmen in den besagten Phasen 2 und 3, die mit dem Modell Kunden gewinnen und Marktanteile erobern wollten, zu unterscheiden.

Das Thema Versicherungsgeschichte ist durch die dreifache Anstrengung von Phillip Hellwege und seinen Mitstreitern mit einem Schlag auf die Bühne der Forschung aufgetreten. Das Unternehmen ist erfolgreich gestartet. Hellwege hat seinen Gegenstand zugleich durch die Betonung des Rechts der Versicherungen aus dem Schatten der rein historischen oder ökonomischen Betrachtung herausgerückt, die bisher vorherrschte. Außerdem ist durch die Konzentration von zwei der drei Bände auf die Tontinen jetzt schon ein zweites wichtiges Ziel des Projekts erreicht. Wenn künftig um die Geschichte des Versicherungsrechts thematisiert wird, wird niemand mehr allein an die Seeversicherung denken.

Funding Open Access funding provided by Projekt DEAL.

Open Access Dieser Artikel wird unter der Creative Commons Namensnennung 4.0 International Lizenz veröffentlicht, welche die Nutzung, Vervielfältigung, Bearbeitung, Verbreitung und Wiedergabe in jeglichem Medium und Format erlaubt, sofern Sie den/die ursprünglichen Autor(en) und die Quelle ordnungsgemäß nennen, einen Link zur Creative Commons Lizenz beifügen und angeben, ob Änderungen vorgenommen wurden.

Die in diesem Artikel enthaltenen Bilder und sonstiges Drittmaterial unterliegen ebenfalls der genannten Creative Commons Lizenz, sofern sich aus der Abbildungslegende nichts anderes ergibt. Sofern das betreffende Material nicht unter der genannten Creative Commons Lizenz steht und die betreffende Handlung nicht nach gesetzlichen Vorschriften erlaubt ist, ist für die oben aufgeführten Weiterverwendungen des Materials die Einwilligung des jeweiligen Rechteinhabers einzuholen.

Weitere Details zur Lizenz entnehmen Sie bitte der Lizenzinformation auf http://creativecommons.org/ licenses/by/4.0/deed.de. 